\title{
Porcine Gastric TFF2 is a Mucus Constituent and Differs from Pancreatic TFF2
}

\author{
René Stürmera Stefan Müller ${ }^{b}$ Franz-Georg Hanisch ${ }^{b}$ Werner Hoffmann ${ }^{a}$ \\ Institute of Molecular Biology and Medicinal Chemistry, Otto-von-Guericke University Magdeburg, \\ Magdeburg, 'bnstitute of Biochemistry II, Medical Faculty, and Central Bioanalytics, Center for Molecular \\ Medicine Cologne, University Köln, Köln, Germany
}

\section{Key Words}

Mucins - Mucus - Pancreas - Stomach - Peptide interactions - TFF peptide - Trefoil factor • Spasmolytic polypeptide

\begin{abstract}
Background/Aims: TFF2 is a secretory peptide (106 amino acid residues) of the gastric mucosa, the porcine exocrine pancreas as well as immune cells and the CNS. It was the aim of this study to compare gastric and pancreatic TFF2. Methods: TFF2 was purified from the porcine stomach and pancreas, respectively, by size-exclusion and anion-exchange chromatography and then analyzed by Western blots as well as mass spectrometry. Results: Gastric and pancreatic TFF2 differed markedly, i.e. gastric TFF2 was exclusively associated with the high molecular mass mucus fraction, whereas pancreatic TFF2 appeared as a low molecular mass product. Unexpectedly, the latter predominantly formed a non-covalently linked homodimer resistant to boiling SDS. In contrast, gastric TFF2 is an integral mucus constituent predominantly binding to the mucin MUC6 in a non-covalent fashion. Conclusion: The non-covalent interaction of TFF2 with the mucin MUC6 is typical of a "link peptide" which is perfectly suited to assemble and stabilize the laminated structure of gastric mucus and to modulate its rheological properties.
\end{abstract}

Copyright @ 2014 S. Karger AG, Basel

\section{Introduction}

TFF2 (originally termed "pancreatic spasmolytic polypeptide") is a secretory peptide of the trefoil factor family (TFF) consisting of 106 amino acid residues [reviews: refs. 1, 2]. It contains two TFF domains, which are stabilized by three highly conserved disulfide bridges each and a seventh disulfide bridge between Cys- 6 and Cys-104; the latter is responsible for a compact circular structure by closing the $\mathrm{N}$ - and C-termini [3-5]. In solution it has been described as a monomer $[4,6]$. TFF2 is an exocrine secretory product of the porcine pancreatic acinar cells as well as the gastric mucosa and Brunner's glands [7]. In human, the major site of expression is the stomach [8] where TFF2 is secreted together with the mucin MUC6 
from cardiac glands, mucous neck cells, and antral gland cells $[9,10]$. There is a dramatic diurnal variation of TFF2 in the human gastric juice [11] and TFF2 is an early response gene after gastric mucosal injury [12]. Pathologically, TFF2 is expressed in the ulcer-associated cell lineage during inflammatory conditions [13] as well as in the metaplastic SPEM lineage [14]. Human TFF2 is N-glycosylated containing a fucosylated N,N'-diacetyllactosediamine oligosaccharide [15]; whereas porcine, murine, and rat TFF2 are devoid of N-glycosylation.

TFF2 is a typical constituent of the layered gastric mucus. In the rat, mouse, and pig two-layered structures have been reported [16-18]; whereas in human the gastric mucus is multi-layered [19]. In the latter, TFF2 is specifically localized within the alternating array of two types of mucins; TFF2 is co-localized with the gland mucin MUC6 and not with the surface mucous cell mucin MUC5AC indicating a physical interaction of TFF2 and MUC6 $[10,20,21]$. Of special note, Helicobacter pylori preferentially colonizes the MUC5AC gel layer [22] probably because the terminal 0-glycans of MUC6 have antimicrobial activity against $H$. pylori [23]. A physical interaction of TFF2 and high molecular mass constituents of mucus (such as mucins) was also observed after size exclusion chromatography (SEC) $[15,24]$. Furthermore, TFF2, and not TFF1 or TFF3, increased dramatically the viscosity and elasticity of porcine gastric mucin solutions in vitro [25]. Similar results were obtained with systemically administered TFF2 in vivo [26]. TFF2-mucin solutions showed non-Newtonian pseudo-plastic behaviour typical of an entangled network [25]. The viscous response was $\mathrm{pH}$-dependent with highest viscosity at low $\mathrm{pH}$ indicating hydrogen bonding [25].

Thus, a physical interaction of TFF 2 with mucus constituents may strengthen the mucus barrier and would explain why only orally administered TFF2 and not parenteral TFF2 protected against induced gastric injury as well as induced colitis in rats [27, 28]. In line with this hypothesis, TFF2 applied luminally to the gastric mucosa inhibited $\mathrm{H}^{+}$permeation through the mucus barrier [29] and Tff2-deficient mice (Tff ${ }^{\mathrm{KO}}$ ) have induced susceptibility to Helicobacter-induced gastritis [30], they showed accelerated progression to dysplasia [31], and their gastric surface $\mathrm{pH}$ was attenuated after photodamage [32]. Thus far, the interaction partners of TFF2 and the type of interaction are not known.

TFF2 is also expressed in the brain and pituitary [33] as well as in immune cells such as macrophages and lymphocytes $[30,34]$. Tff $2^{\mathrm{KO}}$ mice show an altered expression of genes implicated in immune regulation [35] and their immune cells are hyperresponsive to IL-1 $\beta$ stimulation [30].

Also relatively weak motogenic effects have been observed for TFF2 in vitro [compilation: $36]$; this activity is dependent on the ERK1/2 pathway [37, 38]. Furthermore, TFF2 is considered as a low affinity ligand for the chemokine receptor CXCR4 $[39,40]$. However, at present there is a lack of a clear receptor for TFF2. In the past, integrin $\beta 1$ as well as a large membrane glycoprotein with similarity to CRP-Ductin/DMBT1 have been identified as TFF2 binding proteins in the porcine stomach [41]. Here, we systematically compare TFF2 from porcine stomach and pancreas, respectively.

\section{Materials and Methods}

Extraction of proteins from porcine stomach or pancreas

Porcine stomach (antral region) and pancreas were obtained from a local abattoir. Principles of animal care and specific national laws were followed. Extraction of the gastric or pancreatic specimens with a 5 -fold amount (w/v) of buffer was performed as described previously in detail [15].

\section{Protein purification by FPLC}

The gastric or pancreatic extracts (about $10 \mathrm{~mL}$ each) were fractioned by SEC with the ÄKTA ${ }^{\mathrm{TM}}$-FPLC system (Amersham Biosciences, Freiburg, Germany) as described previously [15]. The following columns were used (from GE Healthcare Bio-Sciences AB, Uppsala, Sweden): HiPrep 16/60 Sephacryl S-300 High Resolution (flow rate: $0.5 \mathrm{~mL} / \mathrm{min}, 1.8 \mathrm{~mL}$ fractions) or HiLoad 16/600 Superdex 75 prep grade (flow rate: $1.0 \mathrm{~mL} / \mathrm{min}, 2.0 \mathrm{~mL}$ fractions). Anion-exchange chromatography using a Resource Q6 column (Amersham Biosciences; flow rate: $6.0 \mathrm{~mL} / \mathrm{min}, 1.0 \mathrm{~mL}$ fractions) was performed as described previously [42]. 
SDS-PAGE, SDS-AgGE, and Western blot analysis

Denaturing SDS-PAGE under reducing (5\% $\beta$-mercaptoethanol) or non-reducing conditions, nondenaturing SDS-agarose gel electrophoresis (AgGE) under non-reducing conditions, protein staining with Bio-Safe Coomassie Stain (Bio-Rad Laboratories GmbH, Munich, Germany), silver staining, and periodic acidSchiff (PAS) staining for mucins (dot blot) have been previously described [42]. For special non-reducing SDS-PAGE the concentration of the stacking gel was lowered to 5\%; this enables also detection of very high molecular mass products. Gels obtained after non-reducing SDS-PAGE were subsequently reduced with $1 \%$ B-mercaptoethanol at $50^{\circ} \mathrm{C}$ for $5 \mathrm{~min}$ (post-in-gel reduction).

Western blot analysis after SDS-PAGE, electrophoretic transfer, and fixation with $0.2 \%$ glutaraldehyde were performed according to previous reports [24, 43]. Western blot analysis after SDS-AgGE has been described previously [42] using either capillary blotting or electrophoretic transfer. The porcine mucin MUC6 was detected with the biotinylated lectin GSA-II (from Griffonia simplicifolia II; Biozol Diagnostica Vertrieb GmbH, Eching, Germany) [44] followed by binding of a streptavidin-peroxidase conjugate (Roche Diagnostics GmbH, Mannheim, Germany). The porcine mucin MUC5AC was detected with the polyclonal antiserum PGM2B-1 (44; kindly provided by Prof. J. R. Davies, Malmö). TFF2 was detected with the affinitypurified polyclonal antiserum anti-hTFF2-1 [45]. Bands were visualized with the ECL detection system and semi-quantitative analysis of the bands was performed using the GeneTools software (version 3.07; Syngene, Cambridge, UK) as described in detail [24].

LC-ESI-MS/MS analysis of in-gel digested proteins, database searching

Tryptic digestion of Coomassie stained protein bands and LC-MS/MS analysis have been described in detail previously [42]. Additionally, portions of the Coomassie stained protein band were also digested with chymotrypsin or V8 protease. Trypsin and chymotrypsin digestion were performed in $50 \mathrm{mM}$ ammonium bicarbonate buffer, while $100 \mathrm{mM}$ Tris/HCl pH 8.0 was used with the V8 protease. Extracted peptides of all digests were combined and analyzed in a single run.

Proteins were identified by searching the NCBlnr database (NCBlnr 20130730; 31350673 sequences; 10834990394 residues). Searches were submitted to MASCOT 2.4.0 (Matrix Science Ltd., London, UK) by Proteinscape 3.0 (Bruker Daltoniks, Bremen, Germany) with the enzyme parameter setting "none". Additional setting were: species "Sus scrofa", fixed modifications "carbamidomethyl", optional modifications "methionine oxidation" and "Gln->pyro-Glu". The mass tolerance was set to $0.3 \mathrm{Da}$ for peptide and fragment spectra.

\section{MALDI-TOF/TOF mass spectrometry}

Briefly, a FPLC fraction containing purified TFF2 was passed through a C4 ZipTip (Millipore) equilibrated in $0.1 \%$ TFA. The tip was washed three times with $10 \mu \mathrm{L} 0.1 \%$ TFA. TFF2 was eluted with $10 \mu \mathrm{L}$ $70 \%$ acetonitrile in $0.1 \%$ TFA and mixed $1: 1$ with a saturated solution of sinapinic acid in $50 \%$ acetonitrile, $0.1 \%$ TFA. Protein calibration standard I containing insulin, ubiquitin cytochrome C, and myoglobin (Bruker Daltonics, Bremen, Germany) in 0.1\% TFA was mixed 1:1 with the saturated sinapinic and an equal volume was added to the sample. $1 \mu \mathrm{L}$ of this mixture was applied onto a stainless steel target and linear MALDI mass spectrometry was performed on an UltrafleXtreme MALDI TOF instrument in positive mode (Bruker Daltonics, Bremen, Germany). Singly charged ions corresponding to the standard proteins were used for internal calibration of the mass range from $\mathrm{m} / \mathrm{z} 5734$ to $\mathrm{m} / \mathrm{z} 16952$.

\section{Results}

Characterization of gastric and pancreatic TFF2 by SEC and Western blot analysis

Extracts of porcine stomach or pancreas were subject to SEC (Fig. 1). In gastric extracts, the major amount of TFF2 immunoreactive material was associated with the PAS-positive high molecular mass fraction (fractions 20-24; Fig. 1A). In contrast, TFF2 from pancreatic extracts was eluted with low molecular mass fractions (fractions 51-55; Fig. 1B).

SDS-PAGE under non-reducing conditions and subsequent Western blot analysis of the TFF2-enriched fractions from porcine stomach or pancreas revealed different patterns (Fig. 

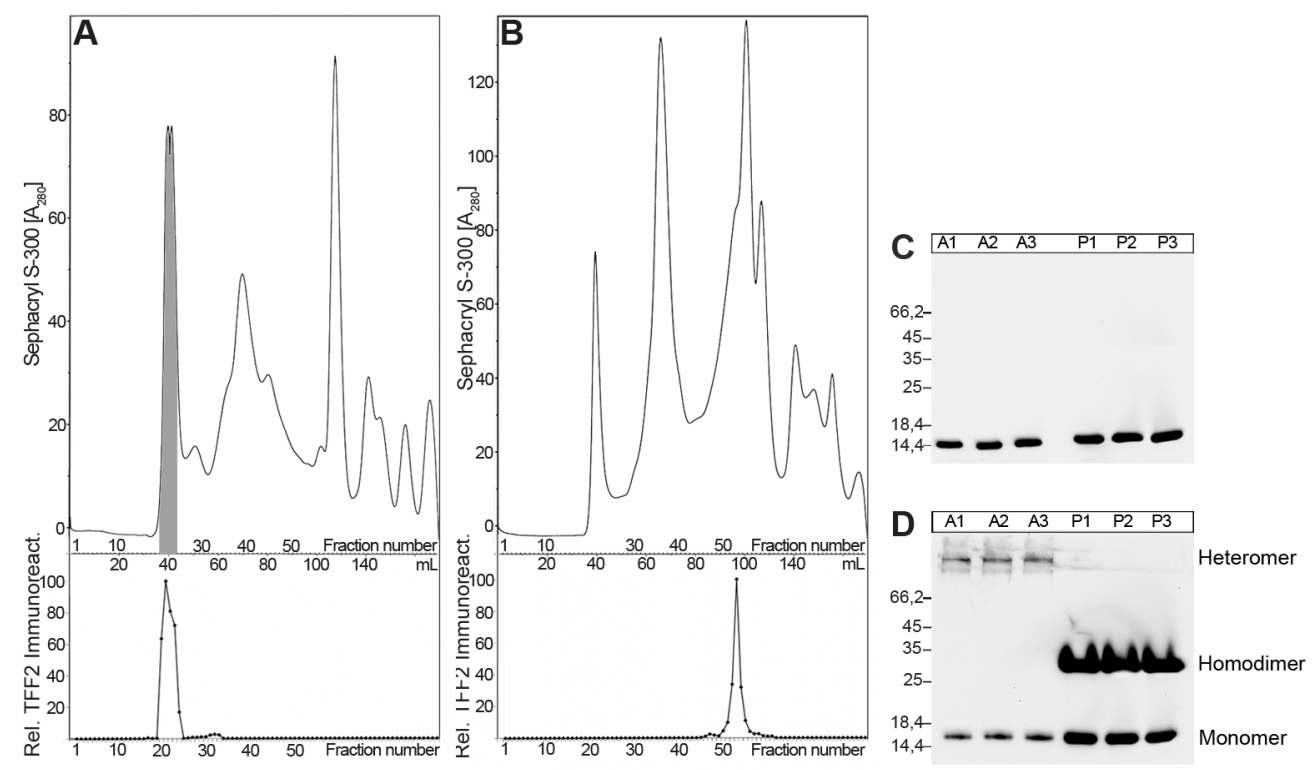

Fig. 1. Comparison of gastric and pancreatic TFF2. (A, B) Size exclusion chromatography of porcine gastric (A) and pancreatic extracts (B), respectively from a single animal and semi-quantitative Western blot analysis. Elution profiles after separation on a Sephacryl S-300 column as determined by absorbance at $280 \mathrm{~nm}$. Fractions positive after PAS staining are shown in grey. Underneath: Distribution of the relative TFF2 immunoreactivity in the fractions as determined by Western blot analysis under reducing conditions and semi-quantitative analysis of the typical 15k-band intensities. (C, D) 15\% SDS-PAGE and subsequent Western blot analysis of TFF2-enriched fractions from porcine gastric and pancreatic extracts after size exclusion chromatography (see A, B). Shown is the TFF2 immunoreactivity. (C) Analysis of antral (A1-A3) and pancreatic (P1-P3) samples from three animals under reducing conditions. (D) Analysis of the antral (A1-A3) and pancreatic (P1-P3) samples under non-reducing conditions (5\% stacking gel; post-in-gel reduction). The molecular mass standard is indicated on the left.

1D). Typical of gastric extracts is TFF2 immunoreactive material in the very high molecular mass region, which hardly enters the stacking gel. In contrast, pancreatic extracts showed a predominant TFF2 immunoreactive band with a $\mathrm{M}_{\mathrm{r}}$ of about $\leq 30 \mathrm{k}$. Furthermore, both gastric and pancreatic extracts contained a TFF2 immunoreactive 16k-band, which probably represents TFF2 monomers. After reduction, only monomeric TFF2 was observed in both gastric and pancreatic extracts (Fig. 1C).

\section{Characterization of TFF2 in the porcine gastric mucus complex}

In order to further analyze the high molecular mass complex of gastric TFF2, an extract of mucus scrapings from the antral porcine mucosa was separated by SEC (Fig. 2A). The major amount of TFF2 immunoreactivity was roughly associated with the PAS-positive high molecular mass fractions (Fig. 2A). Then, the TFF2-positive peak material (fractions 19-21) was subsequently purified by anion-exchange chromatography (Fig. 2B). Of note, the TFF2positive material appeared as a broad peak (fractions 17-41) partly congruent with PASpositive material. These fractions were subsequently separated by SDS-AgGE followed by Western blot analysis for TFF2, MUC5AC, and GSA-II (Fig. 2C). Heterogeneous MUC5AC was mainly found in fractions 18-22; whereas TFF2 was detectable in fractions 16-24 with a $M_{r}$ even higher than that of MUC5AC, hardly entering the gel. A similar pattern was obtained for GSA-II, which recognizes porcine MUC6 [44]. This result is outlined in Fig. 2D by directly comparing Western blot analyses for TFF2, MUC5AC, and GSA-II on the same gel. 

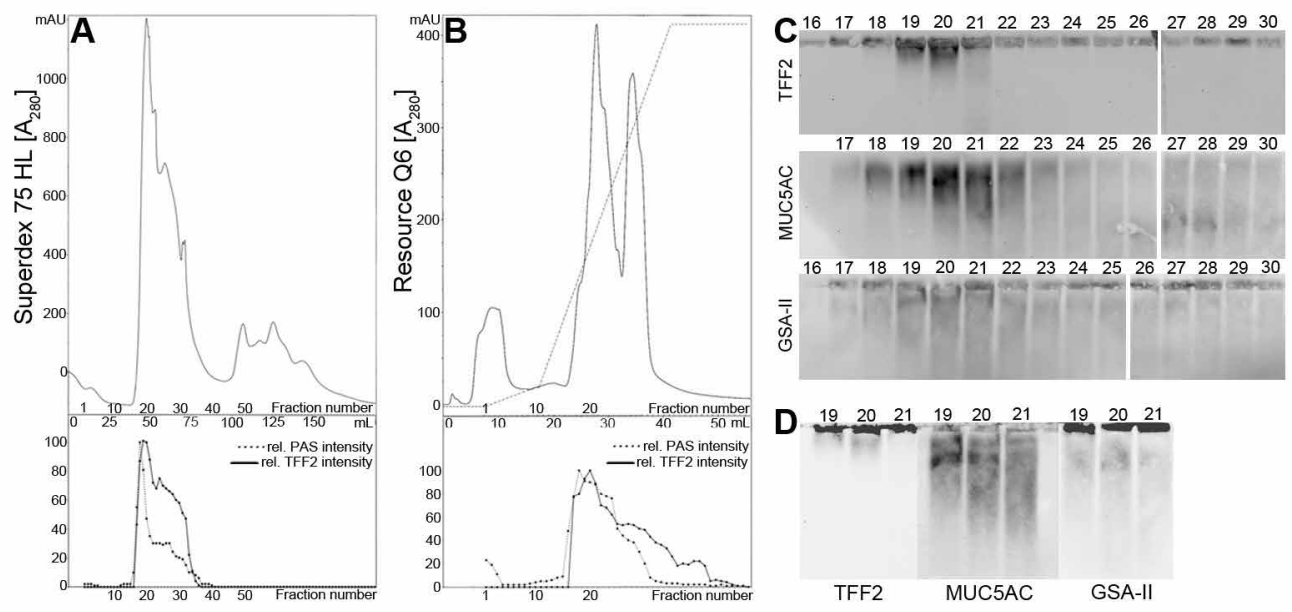

Fig. 2. FPLC purification and analysis of TFF2 from porcine antral mucus scrapings. (A) Elution profile after separation of antral mucus extract from a single animal on a Superdex 75 column as determined by absorbance at $280 \mathrm{~nm}$. Underneath: Distribution of the relative TFF2 immunoreactivity as determined by Western blot analysis under reducing conditions and semi-quantitative analysis of the typical 15k-band intensities (solid line); relative PAS reactivity in the fractions is indicated by a dotted line. (B) Elution profile after separation of the TFF2-positive Superdex 75 fractions No. 19-21 (see A) on a Resource Q6 resin as determined by absorbance at $280 \mathrm{~nm}$. Underneath: Relative TFF2 immunoreactivity (solid line) and relative PAS reactivity (dotted line) of the fractions. (C) 1\% SDS-AgGE and subsequent Western blot analysis of the Resource Q6 fractions No. 16-36 (see B). Shown are the reactivities for TFF2, MUC5AC, and MUC6 (lectin GSA-II). (D) 1\% SDS-AgGE and subsequent Western blot analysis of the Resource Q6 fractions No. 19-21 (see B). Shown is a direct comparison of the reactivities for TFF2, MUC5AC, and MUC6 (lectin GSA-II).

\section{Purification of TFF2 from the porcine pancreas}

Another aim of this study was to purify and identify the novel $\leq 30 \mathrm{k}$ form of pancreatic TFF2. Thus, the pancreatic extract was separated by SEC (Fig. 3A) and the TFF2 positive fractions were subsequently purified by anion-exchange chromatography (Fig. 3B). As a last step, TFF2 positive fractions from the anion-exchange column were again separated by SEC (Fig. 3C). SDS-PAGE under non-reducing conditions showed that the material obtained was very pure and consisted mainly of the $\leq 30 \mathrm{k}$ band and little of the $16 \mathrm{k}$ monomer (Fig. 3D). However, after reduction only the monomeric band was detectable (Fig. 3D). Pancreatic TFF2 purified in this manner was then subject to linear MALDI mass spectrometry as well as LC-ESI-MS/MS analysis.

Characterization of pancreatic TFF2 by LC-ESI-MS/MS analysis and mass spectrometry

The Coomassie stained 15k-band (B15) excised from a reducing gel prepared in parallel to that shown in Fig. 3D (fraction 38) was subjected to in-gel digestion followed by LC-ESIMS/MS for protein identification. The major results are summarized in Table 1. Mature porcine TFF2 was identified with a glutamic acid residue at position 61 (61E, corresponding to $86 \mathrm{E}$ in the precursor) and an alanine residue at position 80 (105A in the precursor).

FPLC-purified TFF2 (fraction 38 from Fig. 3D) was directly analyzed using linear MALDI mass spectrometry to determine the molecular mass of the intact protein. The mass determined using internal calibration markers was 11713.5 (Fig. 3E). 

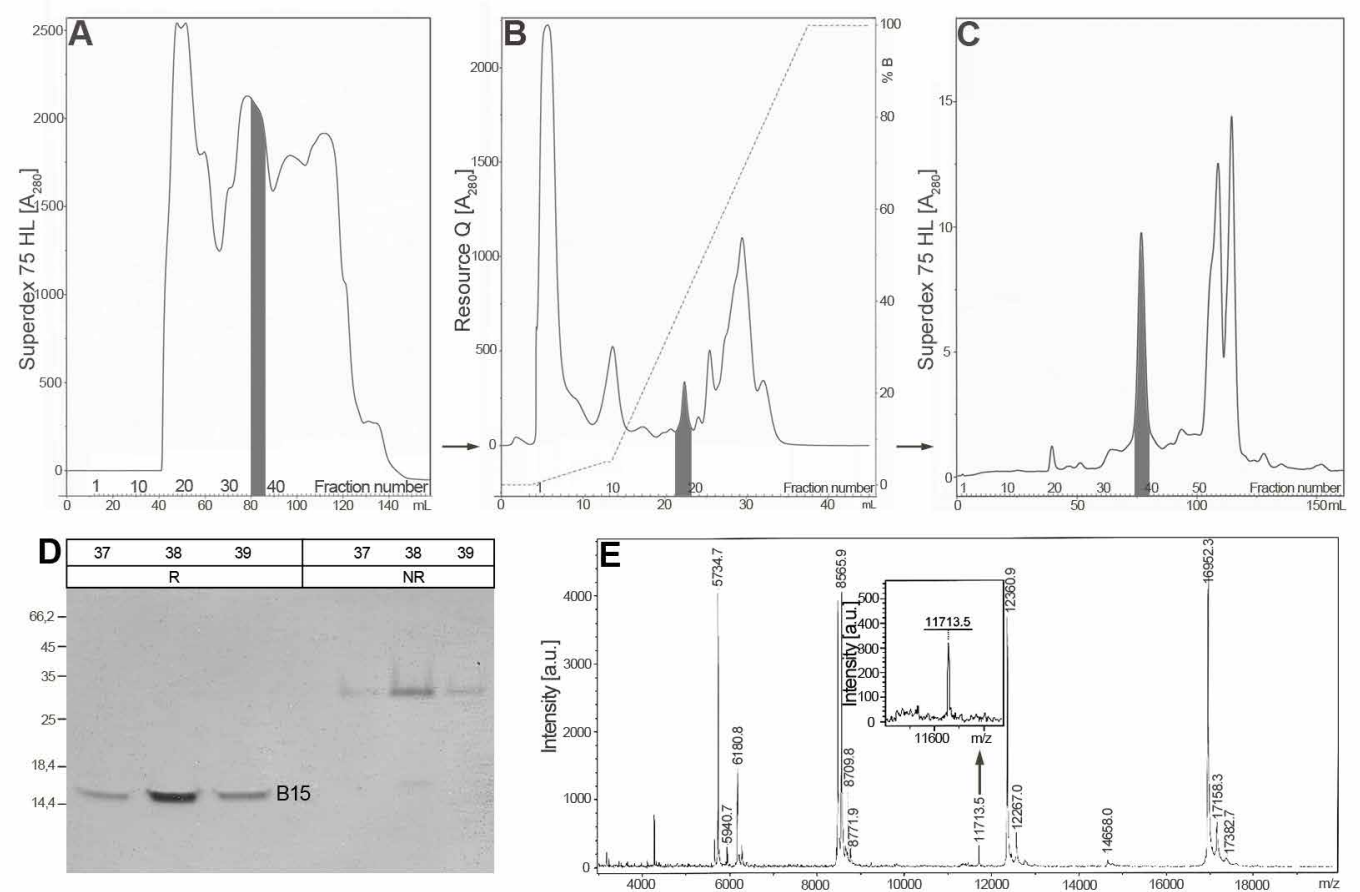

Fig. 3. FPLC purification and analysis of TFF2 from the porcine pancreas of a single animal. (A) Elution profile after separation of pancreatic extract on a Superdex 75 resin as determined by absorbance at $280 \mathrm{~nm}$. TFF2-positive fractions used for further purification are shown in grey. (B) Elution profile after separation of the TFF2-positive Superdex 75 fractions (see A) on a Resource Q6 column as determined by absorbance at $280 \mathrm{~nm}$. The TFF2-positive fractions used for further purification are shown in grey. (C) Elution profile after separation of the TFF2-positive Resource Q6 fractions (see B) on a Superdex 75 resin as determined by absorbance at $280 \mathrm{~nm}$. The TFF2-positive fractions No. 37-39 used for further analysis are shown in grey. (D) 15\% SDS-PAGE of fractions 37-39 (see C) under reducing and non-reducing conditions (post-in-gel reduction) and subsequent silver staining. The molecular mass standard is indicated on the left. (E) MALDI mass spectrometry of purified TFF2 (fraction No. 38 after separation on Superdex 75 HL, see C). The sample was mixed with the calibration standard and analyzed as described in the methods section.

Tab. 1: Proteins identified by peptide mass fingerprint analysis using nano-LC-ESI-MS/MS searching the NCBInr database and detailed protein report for TFF2

\begin{tabular}{lllccc}
\hline Gel Band (in-gel digestion) & $\begin{array}{l}\text { Proteins } \\
\text { Identified }\end{array}$ & $\begin{array}{l}\text { NCBInr } \\
\text { Accession }\end{array}$ & $\begin{array}{c}\text { Nom. Mass } \\
\text { (kDa) }\end{array}$ & $\begin{array}{l}\text { Score } \\
\text { Peptides } \\
\text { Matched }\end{array}$ \\
\hline B15 (trypsin, chymotrypsin, V8) & TFF2 isoform 1 [Sus Scrofa] & gi|335300759 & 14.2 & 208 & 6 \\
& Porcine Trypsin & gil1942351 & 13.3 & 142 & 4 \\
\hline
\end{tabular}

Detailed Protein Report

PREDICTED: trefoil factor 2 isoform 1 [Sus scrofa] (numbering according to the precursor)

\begin{tabular}{|c|c|c|c|c|c|c|c|c|c|c|}
\hline Cmpd. & $\begin{array}{l}\text { No. of } \\
\text { Cmpds. }\end{array}$ & $\mathrm{m} / \mathrm{z}$ meas. & $\begin{array}{l}\Delta \mathrm{m} / \mathrm{z} \\
{[\mathrm{ppm}]}\end{array}$ & $z$ & $\begin{array}{c}\mathbf{R t} \\
\text { [min] }\end{array}$ & Score & $\mathbf{P}$ & Range & Sequence & Modification \\
\hline 60 & 1 & 407.3000 & 232.24 & 2 & 8.2 & 23.2 & 0 & $26-32$ & A.QKPAACR.C & $\begin{array}{l}\text { Carbamidomethyl: 6; Gln->pyro- } \\
\text { Glu: } 1\end{array}$ \\
\hline 375 & 1 & 492.8000 & 115.88 & 2 & 21.5 & 22.8 & 0 & $62-70$ & F.DSQVPGVPW.C & \\
\hline 126 & 1 & 442.2000 & 85.41 & 2 & 12.1 & 37.9 & 0 & $80-86$ & E.SEECVME.V & Carbamidomethyl: 4 \\
\hline 336 & 1 & 818.0000 & 120.56 & 2 & 20.1 & 26.7 & 0 & $87-101$ & E.VSARKNCGYPGISPE.D & Carbamidomethyl: 7 \\
\hline 143 & 2 & 599.0000 & 108.90 & 3 & 12.6 & 45.6 & 0 & $91-106$ & R.KNCGYPGISPEDCAAR.N & Carbamidomethyl: 3,13 \\
\hline 171 & 2 & 833.9000 & 58.71 & 2 & 13.9 & 52.2 & 0 & $92-106$ & K.NCGYPGISPEDCAAR.N & Carbamidomethyl: 2, 12 \\
\hline
\end{tabular}

\section{Discussion}

Porcine pancreatic TFF2 forms a SDS resistant homodimer

As clearly seen after SEC, TFF2 from the porcine pancreas forms a low molecular mass product (Fig. 1B). Surprisingly, pancreatic TFF2 appears on a non-reducing SDS-PAGE mainly 
in the range of $\leq 30 \mathrm{k}$ with a minor band of about $16 \mathrm{k}$, the latter representing the TFF2 monomer (Fig. 1D). After reduction, only monomeric TFF2 was observed (Fig. 1C). Thus, the $\leq 30 \mathrm{k}$ band was expected to represent a TFF2 homodimer, which has not been described in solution thus far. However, porcine TFF2 is well known to crystallize as a non-covalently linked dimer $[4,46,47]$. Generally, a TFF2 dimer is reminiscent to the xP4 peptides from Xenopus laevis stomach containing four TFF domains in tandem [43].

Noteworthy, the $\leq 30 \mathrm{k}$ form of TFF 2 was immunologically detectable on a non-reducing SDS-PAGE only after post-in-gel reduction (Fig. 1D). If the post-in-gel reduction step was omitted, the antibody did not detect this form any more (data not shown). This might be due to the disulfide bridge between cysteine- 6 and cysteine-104 [4], which could mask the antigenic epitope recognized by the antiserum anti-hTFF2-1.

LC-ESI-MS/MS analysis revealed a new TFF2 variant characterized by $61 \mathrm{E}$ and $80 \mathrm{~A}$ in the mature peptide. The $61 \mathrm{E}$ variant is in agreement with the predicted sequence after cDNA cloning [8], but differs from the reported protein sequence (61Q) [3]; whereas 80A is congruent with the reported protein sequence [3], but differs from the sequence predicted from cDNA cloning (80R) [8].

The molecular mass of the $\leq 30 \mathrm{k}$ band was determined to be 11713.5 (Fig. 3E), which is fully compatible with the $61 \mathrm{E} / 80 \mathrm{~A}$ variant of a TFF2 monomer after cyclization of the $\mathrm{N}$-terminal glutamine residue and formation of 7 disulfide bridges (calculated molecular mass: 11713.1). This result indicates that the $\leq 30 \mathrm{k}$ band consists of TFF2 monomers, which non-covalently associate probably to a dimeric form. This is unusual because the noncovalent linkage is partly resistant to boiling SDS during sample preparation for the nonreducing SDS-PAGE (Fig. 1D). However, a similar situation has been described, e.g., for MHC class II molecules [48].

\section{Gastric TFF2 is non-covalently linked to MUC6}

TFF2 from porcine stomach differs markedly from that of the porcine pancreas because it forms a high molecular mass complex (Fig. 1A) as similarly described for human [15, 24].

When the high molecular mass complex of TFF2 was isolated from antral mucosal scrapings (Fig. 2A) and then separated by anion-exchange chromatography (Fig. 2B), the TFF2 positive material was heterogeneous and broadly split between fractions 17 and 41 (Fig. 2B). This is in sharp contrast to pancreatic TFF2, which appeared as a sharp peak (fractions 18/19; Fig. 3B). Thus, gastric TFF2 seemed to be bound to high molecular mass proteins, e.g. mucins, which apparently occur in a broad range of different isoelectric variants probably due to several glycoforms. A systematic analysis of the FPLC fractions by SDS-AgGE clearly revealed that TFF2 occurred mainly in an ultrahigh molecular mass form, which did not even enter the agarose gel (Figs. 2C, 2D). A nearly congruent pattern was obtained for binding of the lectin GSA-II (Figs. 2C, 2D), which specifically recognizes the mucin MUC6 [44]. Thus, TFF2 seems to be predominantly associated with the mucin MUC6. This is in line with the fact that TFF2 and MUC6 are synthesized by the same cells and co-localize within the laminated gastric mucus layer $[9,10,20,21]$. In contrast, the mucin MUC5AC appeared mainly in fractions 18-22 with variable molecular mass considerably lower than that of TFF2 and MUC6 (Figs. 2C, 2D). The lower $\mathrm{M}_{\mathrm{r}}$ of MUC5AC when compared with that of MUC6 is in agreement with previous reports $[44,49]$. Thus, the two TFF domains in TFF2 would be perfectly designated to crosslink MUC6, which might be the reason for the higher $M_{r}$ when compared with MUC5AC.

Under reducing conditions, gastric TFF2 was completely released in monomeric form from the high molecular mass complex (Fig. 1C). In contrast, under non-reducing conditions, two TFF2 forms were observed (Fig. 1D), i.e., monomeric TFF2 as well as a high molecular mass complex, which hardly entered the stacking gel. This indicates that TFF2 is bound to a high molecular mass component (probably MUC6) clearly in a non-covalent fashion and maybe also by a covalent linkage forming a disulfide-linked heteromer. The latter would be reminiscent to that of the TFF1-GKN2 [24, 50] and TFF3-FCGBP heteromers [42]. However, porcine TFF 2 contains an even number of cysteine residues forming seven disulfide bridges; 
thus, at least one disulfide bridge in TFF2 must be opened in order to enable formation of a heteromer. The most likely candidate would be the linkage between Cys- 6 and Cys-104 because this disulfide bridge has been reported to be particular sensitive to reduction with glutathione [51]. As a consequence, TFF2 could then covalently crosslink, e.g. MUC6, either in an intra- or an intermolecular fashion. The latter architecture would establish TFF2 as a covalent link peptide. Alternatively, gastric TFF2 could also interact non-covalently with MUC6 in a manner, which is resistant to boiling SDS similar as the pancreatic TFF2 homodimer. However, both concepts still await further experimental validation.

Generally, the non-covalent and preferential binding of TFF2 to MUC6 could be due to a lectin activity of TFF2 recognizing a specific carbohydrate moiety present in MUC6 but not MUC5AC. For example, the carbohydrate moiety of MUC6, and not MUC5AC, contains the structure GlcNAc $\alpha 1 \rightarrow 4 \mathrm{Gal} \beta 1 \rightarrow \mathrm{R}$ at its non-reducing end [52], which is specifically detected by the lectin GSA-II [44], the monoclonal antibody HIK1083 [52] and the paradoxical concanavalin A staining [53]. Of note, based on its crystal structure a lectin activity has already been predicted for TFF2 enabling the non-covalent cross-linking of mucins [4]. This hypothesis is strongly supported by our results now.

Cross-linking of mucins - both by covalent and non-covalent interactions - is particularly important for the assembly of the laminated structure and the rheological properties of gastric mucus. The latter has already been proven experimentally $[25,26]$. Thus, it is an important goal now to clarify the chemical nature of the non-covalent interaction between TFF2 and MUC6.

\section{Acknowledgements}

The authors thank A. Kohnke and E. Voß for their excellent technical and secretarial assistance, respectively, H. Ragge for his support at the initial phases of this project, Prof. J. R. Davies (Malmö) for providing the PGM2B antiserum, and Dr. J. Lindquist for careful proofreading of the manuscript. MS analyses were performed from the Center for Molecular Medicine Cologne.

\section{References}

1 Thim L: Trefoil peptides: from structure to function. Cell Mol Life Sci 1997;53:888-903.

2 Hoffmann W: TFF Peptides; in Kastin AJ (ed): Handbook of Biologically Active Peptides (2 $2^{\text {nd }}$ edition). San Diego, Elsevier, 2013, pp. 1338-1345.

3 Rose K, Savoy LA, Thim L, Christensen M, Jorgensen KH: Revised amino acid sequence of pancreatic spasmolytic polypeptide exhibits greater similarity with an inducible pS2 peptide found in a human breast cancer cell line. Biochim Biophys Acta 1989;998:297-300.

- Gajhede M, Petersen TN, Henriksen A, Petersen JF, Dauter Z, Wilson KS, Thim L: Pancreatic spasmolytic polypeptide: first three-dimensional structure of a member of the mammalian trefoil family of peptides. Structure 1993;1:253-262.

-5 Carr MD, Bauer CJ, Gradwell MJ, Feeney J: Solution structure of a trefoil-motif-containing cell growth factor, porcine spasmolytic protein. Proc Natl Acad Sci USA 1994;91:2206-2210.

6 Carr MD: ${ }^{1} \mathrm{H}$ NMR-based determination of the secondary structure of porcine pancreatic spasmolytic polypeptide: one of a new family of "trefoil" motif containing cell growth factors. Biochemistry 1992;31:1998-2004.

7 Rasmussen TN, Raaberg L, Poulsen SS, Thim L, Holst JJ: Immunohistochemical localization of pancreatic spasmolytic polypeptide (PSP) in the pig. Histochemistry 1992;98:113-119.

8 Tomasetto C, Rio MC, Gautier C, Wolf C, Hareuveni M, Chambon P, Lathe R: hSP, the domain-duplicated homolog of pS2 protein, is co-expressed with pS2 in stomach but not in breast carcinoma. EMBO J 1990;9:407-414.

-9 Hanby AM, Poulsom R, Singh S, Elia G, Jeffery RE, Wright NA: Spasmolytic polypeptide is a major antral peptide: distribution of the trefoil peptides human spasmolytic polypeptide and pS2 in the stomach. Gastroenterology 1993;105:1110-1116. 
10 Ota H, Hayama M, Momose M, El-Zimaity HMT, Matsuda K, Sano K, Maruta F, Okumura N, Katsuyama T: Colocalization of TFF2 with gland mucous cell mucin in gastric mucous cells and in extracellular mucous gel adherent to normal and damaged gastric mucosa. Histochem Cell Biol 2006;126:617-625.

11 Semple JI, Newton JL, Westley BR, May FEB: Dramatic diurnal variation in the concentration of the human trefoil peptide TFF2 in gastric juice. Gut 2001;48:648-655.

12 Wong WM, Playford RJ, Wright NA: Peptide gene expression in gastrointestinal mucosal ulceration: ordered sequence or redundancy? Gut 2000;46:286-292.

13 Wright NA: Aspects of the biology of regeneration and repair in the human gastrointestinal tract. Philos Trans R Soc Lond B 1998;353:925-933.

14 Schmidt PH, Lee JR, Joshi V, Playford RJ, Poulsom R, Wright NA, Goldenring JR: Identification of a metaplastic cell lineage associated with human gastric adenocarcinoma. Lab Invest 1999;79:639-646.

15 Hanisch F-G, Ragge H, Kalinski T, Meyer F, Kalbacher H, Hoffmann W: Human gastric TFF2 peptide contains an N-linked fucosylated N,N'-diacetyllactosediamine (LacdiNAc) oligosaccharide. Glycobiology 2013;23:211.

16 Taylor C, Allen A, Dettmar PW, Pearson JP: Two rheologically different gastric mucus secretions with different putative functions. Biochim Biophys Acta 2004;1674:131-138.

17 Phillipson M, Johansson MEV, Henriksnäs J, Petersson J, Gendler SJ, Sandler S, Persson AEG, Hansson GC, Holm L: The gastric mucus layers: constituents and regulation of accumulation. Am J Physiol 2008;295:G806-812.

18 Ermund A, Schutte A, Johansson ME, Gustafsson JK, Hansson GC: Studies of mucus in mouse stomach, small intestine, and colon. I. Gastrointestinal mucus layers have different properties depending on location as well as over the Peyer's patches. Am J Physiol 2013;305:G341-347.

19 Ota H, Katsuyama T: Alternating laminated array of two types of mucin in the human gastric surface mucous layer. Histochem J 1992;24:86-92.

20 Suzuki K, Hayama M, Nakamura M, Yamauchi K, Maruta F, Miyagawa S, Ota H: Trefoil factor 2 in gland mucous cell mucin in the mucous gel covering normal or damaged gastric mucosa using the Mongolian gerbil model. Scand J Gastroenterol 2006;41:1390-1397.

-21 Kubota S, Yamauchi K, Sugano M, Kawasaki K, Sugiyama A, Matsuzawa K, Akamatsu T, Ohmoto Y, Ota H: Pathophysiological investigation of the gastric surface mucous gel layer of patients with Helicobacter pylori infection by using immunoassays for trefoil factor family 2 and gastric gland mucous cell-type mucin in gastric juice. Dig Dis Sci 2011;56:3498-3506.

-22 Hidaka E, Ota H, Hidaka H, Hayama M, Matsuzawa K, Akamatsu T, Nakayama J, Katsuyama T: Helicobacter pylori and two ultrastructurally distinct layers of gastric mucous cell mucins in the surface mucous gel layer. Gut 2001;49:474-480.

23 Kawakubo M, Ito Y, Okimura Y, Kobayashi M, Sakura K, Kasama S, Fukuda MN, Fukuda M, Katsuyama T, Nakayama J: Natural antibiotic function of a human gastric mucin against Helicobacter pylori infection. Science 2004;305:1003-1006.

-24 Kouznetsova I, Laubinger W, Kalbacher H, Kalinski T, Meyer F, Roessner A, Hoffmann W: Biosynthesis of gastrokine-2 in the human gastric mucosa: restricted spatial expression along the antral gland axis and differential interaction with TFF1, TFF2 and mucis. Cell Physiol Biochem 2007;20:899-908.

25 Thim L, Madsen F, Poulsen SS: Effect of trefoil factors on the viscoelastic properties of mucus gels. Eur J Clin Invest 2002;32:519-527.

26 Kjellev S, Nexo E, Thim L, Poulsen SS: Systemically administered trefoil factors are secreted into the gastric lumen and increase the viscosity of gastric contents. Br J Pharmacol 2006;149:92-99.

27 Babyatsky MW, deBeaumont M, Thim L, Podolsky DK: Oral trefoil peptides protect against ethanol- and indomethacin-induced gastric injury in rats. Gastroenterology 1996;110:489-497.

28 Poulsen SS, Kissow H, Hare K, Hartmann B, Thim L: Luminal and parenteral TFF2 and TFF3 dimer and monomer in two models of experimental colitis in the rat. Regul Pept 2005;126:163-171.

29 Tanaka S, Podolsky DK, Engel E, Guth PH, Kaunitz JD: Human spasmolytic polypeptide decreases proton permeation through gastric mucus in vivo and in vitro. Am J Physiol 1997;272:G1473-1480.

-30 Kurt-Jones EA, Cao L, Sandor F, Rogers AB, Whary MT, Nambiar PR, Cerny A, Bowen G, Yan J, Takaishi S, Chi AL, Reed G, Houghton J, Fox JG, Wang TC: Trefoil family factor 2 is expressed in murine gastric and immune cells and controls both gastrointestinal inflammation and systemic immune responses. Infect Immun 2007;75:471-480. 
-31 Fox JG, Rogers AB, Whary MT, Ge Z, Ohtani M, Jones EK, Wang TC: Accelerated progression of gastritis to dysplasia in the pyloric antrum of TFF $\%$ C57BL6 x Sv129 Helicobacter pylori-infected mice. Am J Pathol 2007;171:1520-1528.

- 32 Xue L, Aihara E, Podolsky DK, Wang TC, Montrose MH: In vivo action of trefoil factor 2 (TFF2) to speed gastric repair is independent of cyclooxygenase. Gut 2010;59:1184-1191.

-33 Hinz M, Schwegler H, Chwieralski CE, Laube G, Linke R, Pohle W, Hoffmann W: Trefoil factor family (TFF) expression in the mouse brain and pituitary: changes in the developing cerebellum. Peptides 2004;25:827832.

34 Cook GA, Familari M, Thim L, Giraud AS: The trefoil peptides TFF2 and TFF3 are expressed in rat lymphoid tissues and participate in the immune response. FEBS Lett 1999;456:155-159.

-35 Baus-Loncar M, Kayademir T, Takaishi S, Wang T: Trefoil factor family 2 deficiency and immune response. Cell Mol Life Sci 2005;62:2947-2955.

-36 Hoffmann W, Jagla W: Cell type specific expression of secretory TFF peptides: colocalization with mucins and synthesis in the brain. Int Rev Cytol 2002;213:147-181.

- 37 Graness A, Chwieralski CE, Reinhold D, Thim L, Hoffmann W: Protein kinase C and ERK activation are required for TFF-peptide-stimulated bronchial epithelial cell migration and tumor necrosis factor- $\alpha$ induced interleukin-6 (IL-6) and IL-8 secretion. J Biol Chem 2002;277:18440-18446.

-38 Chwieralski CE, Schnurra I, Thim L, Hoffmann W: Epidermal growth factor and trefoil factor family 2 synergistically trigger chemotaxis on BEAS-2B cells via different signaling cascades. Am J Respir Cell Mol Biol 2004;31:528-537.

-39 Dubeykovskaya Z, Dubeykovskiy A, Solal-Cohen J, Wang TC: Secreted trefoil factor 2 activates the CXCR4 receptor in epithelial and lymphocytic cancer cell lines. J Biol Chem 2009;284:3650-3662.

40 Hoffmann W: Trefoil factor family (TFF) peptides and chemokine receptors: A promising relationship. J Med Chem 2009;52:6505-6510.

$\checkmark 41$ Thim L, Mortz E: Isolation and characterization of putative trefoil peptide receptors. Regul Pept 2000;90:61-68.

-42 Albert TK, Laubinger W, Müller S, Hanisch FG, Kalinski T, Meyer F, Hoffmann W: Human intestinal TFF3 forms disulfide-linked heteromers with the mucus-associated FCGBP protein and is released by hydrogen sulfide. J Proteome Res 2010;9:3108-2117.

43 Jagla W, Wiede A, Kölle S, Hoffmann W: Differential expression of the TFF-peptides XP1 and xP4 in the gastrointestinal tract of Xenopus laevis. Cell Tissue Res 1998;291:13-18.

44 Nordman H, Davies JR, Carlstedt I: Mucus glycoproteins from pig gastric mucosa: Different mucins are produced by the surface epithelium and the glands. Biochem J 1998;331:687-694.

45 Jagla W, Wiede A, Dietzmann K, Rutkowski K, Hoffmann W: Co-localization of TFF3 peptide and oxytocin in the human hypothalamus. FASEB J 2000;14:1126-1131.

46 De A, Brown DG, Gorman MA, Carr M, Sanderson MR, Freemont PS: Crystal structure of a disulfide-linked "trefoil" motif found in a large family of putative growth factors. Proc Natl Acad Sci USA 1994;91:10841088.

-47 Petersen TN, Henriksen A, Gajhede M: Structure of porcine pancreatic spasmolytic polypeptide at $1.95 \mathrm{~A}$ resolution. Acta Cryst D 1996;52:730-737.

48 Neefjes JJ, Ploegh HL: Inhibition of endosomal proteolytic activity by leupeptin blocks surface expression of MHC class II molecules and their conversion to SDS resistant $\alpha ß$ heterodimers in endosomes. EMBO J 1992;11:411-416.

-49 Nordman H, Davies JR, Lindell G, de Bolos C, Real F, Carlstedt I: Gastric MUC5AC and MUC6 are large oligomeric mucins that differ in size, glycosylation and tissue distribution. Biochem J 2002;364:191-200.

-50 Westley BR, Griffin SM, May FEB: Interaction between TFF1, a gastric tumor suppressor trefoil protein, and TFIZ1, a brichos domain-containing protein with homology to SP-C. Biochemistry 2005;44:7967-7975.

-51 Otto WR, Rao J, Cox HM, Kotzian E, Lee CY, Goodlad RA, Lane A, Gorman M, Freemont PA, Hansen HF, Pappin D, Wright NA: Effects of pancreatic spasmolytic polypeptide (PSP) on epithelial cell function. Eur J Biochem 1996;235:64-72.

-52 Ishihara K, Kurihara M, Goso Y, Urata T, Ota H, Katsuyama T, Hotta K: Peripheral $\alpha$-linked $\mathrm{N}$-acetylglucosamine on the carbohydrate moiety of mucin derived from mammalian gastric gland mucous cells: epitope recognized by a newly characterized monoclonal antibody. Biochem J 1996;318:409-416.

53 Nakayama J, Katsuyama T, Fukuda M: Recent progress in paradoxical concanavalin A staining. Acta Histochem Cytochem 2000;33:153-157. 